\title{
Programa de inclusión para actores de Educación Superior Técnico-Profesional
}

\author{
Marcela Pérez Poouet \\ mperez@virginiogomez.cl \\ Instituto Profesional Virginio Gómez \\ Paulina Muñoz Villalobos \\ Instituto Profesional Virginio Gómez \\ Victoria Aravena Rivas \\ Instituto Profesional Virginio Gómez
}

\section{Resumen}

El Programa de Inclusión del Instituto Profesional Virginio Gómez ha implementado políticas que tienen como objetivo generar una cultura inclusiva a través de acciones que favorecen el ingreso, permanencia y egreso de los estudiantes en situación de discapacidad, concretando ajustes necesarios en el proceso de admisión, generando instancias que fomentan el acceso a la información de planes y programas -de sus respectivas carreras en condiciones de equidad-, realizando difusión y concienciación intra- y extrainstitucional, educando desde una perspectiva inclusiva y modificando la infraestructura en términos de accesibilidad universal.

Algunas barreras en el ingreso de los estudiantes están relacionadas con falta de información sobre el proceso académico. Por otra parte, se percibe en los docentes la necesidad de contar con conocimientos, herramientas y estrategias que promuevan la inclusión educativa y diseño universal de aprendizaje dentro del aula.

Esta innovación ha sido implementada desde el año 2017, buscando que los estudiantes en situación de discapacidad finalicen su proceso académico y no abandonen sus carreras. A la fecha, se han matriculado, a nivel institucional, 34 estudiantes con discapacidad física y sensorial, contando con una retención de un $79 \%$, que responde a los indicadores institucionales. A partir de esta innovación, el Instituto puede identificar a los estudiantes con discapaci- 
dad y originar los mecanismos de acompañamiento necesarios y acciones pertinentes que suman a todos los actores clave de la comunidad, lo cual favorece la experiencia y la progresión académica.

Palabras clave: cultura inclusiva, educación superior, discapacidad, progresión académica.

\section{Abstract}

The inclusion program of Virginio Gómez Professional Institute has implemented policies use with the aim to generate an Inclusive Culture, through actions that favor the income, permanence and egress of students with disabilities, by making necessary adjustments in the admission process, by promoting access to degree plans and programs information under equitable conditions, raising institutional awareness, educating the community from a inclusive perspective and changing the infrastructure in terms of Universal Accessibility.

Some student barriers to access are related by the lack of information of the academic process. Teachers must have the knowledge, tools and strategy that promote an inclusive education and the universal design of learning inside the classroom.

Some student barriers to access are related by the lack of information of the academic process. Teachers must have the knowledge, tools and strategy that promote an inclusive education and the universal design of learning inside the classroom.

This innovation has been implemented since 2017, looking for students with disabilities complete their education and stay there. To date has been enrolled to professional institute, 34 students with physical and sensorial disability, with $79 \%$ of retention that correspond to the institutional indicators. From this the Institute can identify students with disabilities and generate the mechanisms of necessary accompaniment and relevant measures which includes every member of the community, facilitating the experience and academic progression.

Key words: inclusive culture, higher education, disability, academic progress.

\section{Problema y justificación del estudio}

Desarrollar una cultura institucional inclusiva es el desafío de innovación que asume el Instituto Profesional Virginio Gómez, con la finalidad de dar respuestas a los estudiantes en situación de discapacidad que ingresan a la educación superior, favorecien- 
do su ingreso, permanencia y egreso a través de cambios a nivel de gestión como académicos.

Los primeros objetivos planteados en esta innovación fueron:

- Diseñar e implementar un programa de inclusión integral, que contribuya a la retención, titulación e inserción laboral de estudiantes en situación de discapacidad.

- Favorecer el aprendizaje significativo y desarrollo de competencias de estudiantes en situación de discapacidad, a través de la incorporación de nuevas metodologías que permitan atender necesidades específicas.

Posteriormente, se desarrollaron otras actividades que facilitan la instalación de una cultura inclusiva en la comunidad académica: charlas y reuniones de concienciación con actores internos y externos de la comunidad; implementación de un modelo de diseño universal de aprendizaje; capacitaciones en lengua de señas chilena; articulación con liceos, empresas e instituciones ligadas a la discapacidad de la región del Biobío y Ñuble.

De acuerdo con el II Estudio Nacional de la Discapacidad (ENDISC II) del año 2015, el $20 \%$ de la población adulta en Chile (de 18 años y más) se encuentra en situación de discapacidad. Junto con esto, se señala una mayor prevalencia en los quintiles de menores ingresos $(25,5 \%$ para el primer quintil y un $24,4 \%$ para el segundo). De la totalidad de la población adulta en situación de discapacidad, solo un 9,1 \% ha finalizado estudios de educación superior, en contraste con la población adulta sin discapacidad, que alcanza el $20 \%$. Esto se explicaría por una serie de barreras que experimenta este grupo para acceder, permanecer y culminar sus carreras de educación superior (Errandonea, 2016: 5). En el Informe sobre la Educación Superior en América Latina y el Caribe (año 2006), del Instituto Internacional de la Unesco para la Educación Superior en América Latina y el Caribe (IESALC), si bien se reconoce el trabajo realizado por las Instituciones de Educación Superior sobre esta materia, de igual forma se mencionan dichas barreras: carencia de políticas institucionales -a pesar del reconocimiento de la legislación internacional y de contar con una legislación nacional que garantiza los derechos de las personas con discapacidad-; persistencia de las dificultades en el acceso y permanencia, por la escasa voluntad 
política de las autoridades de las instituciones de educación superior; barreras arquitectónicas, pedagógicas y comunicacionales; estas dos últimas causadas por el tipo de formación profesional de los docentes de educación superior, quienes no cuentan con formación pedagógica inicial y cuya enseñanza se centra en el tecnicismo más que en la didáctica, dificultando muchas veces la implementación de adecuaciones curriculares no significativas, y barreras actitudinales que involucran componentes cognitivos, afectivos y conductuales y que son reconocidas como las más difíciles de cambiar; insuficiente uso de tecnologías de la información y las comunicaciones (TIC); predominio de programas y servicios asistencialistas, sin participación de otros actores clave ni de los estudiantes con capacidades diferentes; falta de registros estadísticos que proporcionen información sobre estudiantes, docentes y funcionarios con discapacidad, entre otras.

Por lo anterior, y dada la gran cantidad de declaraciones y otros documentos del sistema interamericano y ámbito iberoamericano que sostienen la inclusión de personas con discapacidad en la educación superior, es que, en el año 2010, Chile promulga la Ley 20.422, que señala, entre otras cosas, que «las instituciones de educación superior deberán contar con mecanismos que faciliten el acceso de las personas con discapacidad...». Aunque, de acuerdo con la Unesco (2006), en la legislación se otorga una atención menor a las estrategias que favorecen la permanencia y el egreso, en nuestro país este hito ha sido considerado un sustento importante en el que se fundamentan las acciones que las Instituciones de Educación Superior llevan a cabo para trabajar sobre estas barreras, algunas de las cuales están comprometidas con esta materia desde hace bastante tiempo.

Según Lissi, Zuzulich, Salinas, Achiardi, Hojas y Pedrals (2009), las instituciones de educación superior inclusivas deben contar con políticas que promuevan la inclusión y la incorporación de procesos que favorezcan el acceso, permanencia y egreso, actividades permanentes de sensibilización y concienciación en la comunidad educativa, integrando medidas antidiscriminatorias. Todo esto contribuye a generar una cultura inclusiva.

Por esta razón, el objetivo de la presente publicación es dar a conocer las acciones que ha implementado el Programa de Inclusión del Instituto Profesional Virginio Gómez, con la finalidad de favorecer la consecución de lo señalado anteriormente. 


\section{Marco teórico}

Hablar de inclusión en la educación superior requiere, más que instalar procesos, generar una cultura inclusiva que permita descubrir los beneficios que trae la diversidad a un sistema. Para esto es importante entender la inclusión de las personas en situación de discapacidad desde un modelo social, el cual considera que «las causas que originan la discapacidad no son religiosas ni científicas, sino que son, en gran medida, sociales» (Victoria, 2013).

Esta nueva forma de pensar se enmarca en los derechos humanos de las personas con discapacidad, celebrados el año 2006 en la Convención de la Organización de las Naciones Unidas sobre los Derechos de las Personas con Discapacidad, donde este concepto no se centra en la persona y su carencia, como hace el modelo médico, sino en su interacción con el entorno y en las dificultades que este le presenta para una plena participación. Por tanto, las soluciones para las personas con discapacidad no deben centrarse en lo individual, sino que deben estar dirigidas a la sociedad. Este modelo de derechos humanos pone el énfasis en garantizar el respeto de la dignidad humana, igualdad y la libertad personal, propiciando la inclusión social, sobre la base de los siguientes principios: autonomía personal, no discriminación, accesibilidad universal, normalización del entorno, diálogo civil, entre otros.

El modelo social concentra su atención en descubrir las habilidades y capacidades que tienen las personas en situación de discapacidad, con la finalidad de potenciarlo involucrando en este proceso a su entorno inmediato y posteriormente otros entornos. Para lo anterior se requiere un proceso socializador y concienciador, con la finalidad de educar y desde ahí trabajar las ideas, prejuicios o sentimientos que se tengan sobre la discapacidad. Finalmente, percibe el entorno como facilitador de oportunidades en términos de la equidad y la eliminación de barreras (Victoria, 2013).

En el Estado de Yucatán (México) se ha impulsado la expedición de la Ley para Prevenir y Eliminar la Discriminación, en la que se reconocen los derechos a la igualdad y no discriminación y la accesibilidad universal de las personas con discapacidad, así como la promulgación del reglamento respectivo, con lo que se 
abre el camino a la instauración de políticas públicas eficaces para este sector poblacional (Victoria, 2013).

En algunos estudios que promueven culturas inclusivas, como el de la Universidad de Murcia (España), denominado Diversidad e inclusión educativa: algunas reflexiones sobre el liderazgo escolar, se pone el énfasis en la importancia en los sistemas educativos del liderazgo en los procesos de inclusión educativa, ya que la organización educativa es responsable de implementar principios que favorezcan una cultura inclusiva permitiendo iniciar, sostener y evaluar los cambios organizacionales y didácticos de la inclusión educativa.

Otro estudio realizado en Valencia (España), Una escuela de todas (las personas) para todas (las personas), nos invita a conocer su experiencia de más de veinticinco años, donde ha realizado y consolidado auténticos y profundos valores y prácticas escolares inclusivas. Su desafío consistió en asumir riesgos que respondieran a su visión educativa, sus prácticas innovadoras en las formas de enseñar con la finalidad de hacer posible el aprendizaje y la participación de una diversidad de alumnos(as) lo que nos hace pensar y ver que «es posible» lo que anhelamos (Echeita y Duk, 2008).

La cultura inclusiva en los espacios educativos solo será posible si se apoyan los procesos de cambio y de adaptación que son necesarios para dar una respuesta positiva a la diversidad. Por ende, uno de sus desafíos será que sus profesionales favorezcan en el aula una cultura de respeto a la diversidad (Gutiérrez-Ortega, Martín-Cilleros y Jenaro-Ríos, 2018).

El acceso, la participación y los logros de los estudiantes son elementos que debe considerar la inclusión educativa, lo cual implica transformar la cultura, las políticas y las prácticas de los establecimientos educacionales con la finalidad de dar respuesta a la diversidad (Plancarte, 2017).

La Unesco (2009) considera a la educación inclusiva como una estrategia clave para la "Educación para Todos», partiendo de la base de que la educación es un derecho humano básico y fundamental de una sociedad más justa e igualitaria.

La inclusión considera el modelo social, el cual tiene como premisa la aceptación de la diversidad, considerando que es el sistema el que debe responder al estudiante y no a la inversa que era lo que sucedía con el modelo médico. La educación debe ba- 
sarse en la diversidad, respetando y considerando las diferencias tanto personales como psicológicas o sociales, lo que implica un cambio de visión de la educación en sus procesos de enseñanza y aprendizaje (Plancarte, 2017).

Echeita y Ainscow (2011), tras varias investigaciones y trabajos, consideran que la inclusión debe abarcar la totalidad de los procesos y las políticas y a todos los estudiantes que puedan ser objeto de exclusión. Para lo anterior, señalan cuatro elementos clave que se deben considerar en cualquier definición de inclusión educativa: 1) la inclusión es un proceso, es aprender a vivir con las diferencias y cómo aprender de las diferencias; 2) las diferencias son vistas positivamente para fortalecer el aprendizaje; 3) la inclusión se relaciona con la identificación y eliminación de barreras para el aprendizaje, por lo que implica recopilar, cotejar y evaluar la información de la gran variedad de recursos, para planificar las mejoras en las políticas y prácticas, y 4) la inclusión se refiere a la presencia, participación y logros de todos los estudiantes.

El concepto de educación inclusiva se basa en un principio de «equidad» en pos de la igualdad futura mediante un proceso lineal. (Armijo-Cabrera, 2018)

En la Declaración de Salamanca de 1994 se plantea que los sistemas educativos deben apuntar a la diversidad, es decir, transformarse en sistemas inclusivos respondiendo a la necesidad de educar a todos los niños y niñas de su comunidad:

Las prestaciones educativas especiales son un problema que afecta por igual a los países del norte y a los del sur, que no pueden progresar aisladamente, sino que deben formar parte de una estrategia global de la educación y, desde luego, de nuevas políticas sociales y económicas. Esto requiere una reforma considerable de la escuela ordinaria. (Declaración de Salamanca, 1994, extraído de Parra, 2011)

La educación inclusiva es para todos, valora la diversidad y entiende que todos los alumnos participan de un único sistema escolar, proporcionando un currículo apropiado que responda a sus intereses y necesidades. 
El término diversidad hace alusión a las diferencias que existen entre todas las personas, y no solamente a quienes tienen alguna discapacidad. La inclusión así implica en que todas las personas de la escuela aprenden a vivir con las diferencias y además a aprender de las diferencias. (Mel Ainscow, 2001, citado por Parra, 2011)

Se entiende la inclusión como un proceso que busca fomentar el respeto por los derechos humanos y las libertades fundamentales (Vásquez y Alarcón, 2016). La inclusión debe ser participativa y transformadora, fomentando así la no discriminación de grupos minoritarios por causa de sus diferencias (Parra, 2011). Esto implica valorar a cada persona como tal (Paz, 2018). Para ello, un componente importante de la cultura inclusiva en un centro educativo son sus valores. Se consideran «junto con las concepciones, la pauta que da sentido de dirección, manteniendo las culturas, políticas y prácticas dirigidas hacia la inclusión» (Gutiérrez-Ortega, Martín-Cilleros y Jenaro-Ríos, 2018).

Chile, a partir de la Ley 20.422 del año 2010, ratifica lo propuesto por la Unesco, en la Convención Internacional para la Eliminación de todas las Formas de Discriminación contra las personas con Discapacidad (1999). Esta ley genera lineamientos para la igualdad de oportunidades e inclusión social, estableciendo normas para la educación superior.

\section{Metodología de investigación}

El presente artículo está orientado a describir la innovación desarrollada por el Programa de Inclusión del Instituto Profesional Virginio Gómez, desde su diseño, en el año 2016, hasta su implementación, desde el año 2017 hasta la fecha, y analizar sus resultados cuantitativos y cualitativos.

\subsection{Descripción del contexto}

El Instituto Profesional Virginio Gómez es una institución perteneciente a la Universidad de Concepción, con sedes en Concepción, Los Ángeles y Chillán, y cuenta con carreras técnicas y profesionales impartidas en jornadas diurna y vespertina. De sus estudiantes, sobre el 90 \% tienen una situación económica solo 
suficiente e insuficiente, factor que se relaciona con una mayor cantidad de personas en situación de discapacidad, de acuerdo con la ENDISC II. Recibe todos los años a estudiantes con distintas situaciones de discapacidad, información que se obtiene de la encuesta de caracterización que se aplica desde el año 2014. Frente a esta situación, se comienza a diseñar en el año 2016 un Programa, bajo el alero del Departamento de Apoyo al Estudiante, cuyo objetivo es instalar una cultura inclusiva en la institución, en conjunto con distintos actores clave, intra y extrainstitucionales, para favorecer el ingreso, permanencia y egreso de estos estudiantes. Es así como se realizan diversas acciones que conllevan ajustes en los procesos de admisión, en el ámbito académico, a través de adecuaciones curriculares y diseño universal para el aprendizaje, de infraestructura y jornadas de difusión para educar desde una perspectiva inclusiva, entre otras.

\subsection{Participantes}

El Programa de Inclusión nace en el Departamento de Apoyo al Estudiante, el que, en conexión con instituciones y empresas externas y con la colaboración de los departamentos de Admisión y Comunicaciones, Análisis Institucional e Informática, Desarrollo Curricular, Desarrollo Docente, Formación Transversal, Directores de Carrera, docentes y los estudiantes en situación de discapacidad de la institución se han encargado de diseñar, ejecutar y evaluar las distintas acciones implementadas.

\subsection{Instrumentos y procedimientos}

Para el diseño del Programa de Inclusión se realizaron encuentros con 11 alumnos de la cohorte 2016 que señalaron en Encuesta de Caracterización estar inscritos en el Registro Nacional de Discapacidad. Estos encuentros se iniciaron en junio de 2016 en las tres sedes de la institución y tuvieron como objetivo realizar un levantamiento de las necesidades que en ese momento percibían estos alumnos en el Instituto, en relación con su discapacidad. Para ello se elaboró una Pauta de Entrevista Individual. Paralelamente se consultó bibliografía actualizada y normativa vigente, se conocieron experiencias de instituciones de educación superior nacionales e internacionales y se efectuaron reuniones 
de trabajo con los distintos departamentos del instituto del área académica. Con la información obtenida se definen los principios que guiarán la implementación del programa de inclusión (corresponsabilidad, educación y concienciación, cultura inclusiva, modelo social y perspectiva de derecho) y las acciones desarrolladas, que son:

- Difusión intra- y extra institucional: se realizan charlas y reuniones reflexivas con la comunidad educativa y liceos, instituciones ligadas a la temática de discapacidad y empresas externas, con la finalidad de dar un espacio de reflexión y análisis respecto de los conceptos de discapacidad e inclusión social.

- Enseñanza desde la diversidad: se incorpora esta temática en asignaturas del Departamento de Formación Transversal, las cuales son: Cultura y Sociedad, Comunicación y Aprendizaje, y Taller de Estrategias de Emprendimiento y Empleabilidad.

- Ajustes necesarios en el proceso de admisión: se fortalece el proceso de admisión institucional, integrando el concepto de Inclusión para detectar, identificar y orientar a los alumnos en situación de discapacidad, a través de distintos medios: capacitar en temática de inclusión a los encargados de matrícula, implementación de mecanismos de acceso en página web institucional y de detección en plataformas informáticas.

- Formación desde una perspectiva inclusiva: se elabora el Manual de Atención a la Discapacidad con la colaboración del Departamento de Desarrollo Curricular, a partir de revisiones bibliográficas y considerando lo declarado en el Proyecto Educativo Institucional, con la finalidad de que todos los docentes puedan consultarlo a través de la intranet institucional. Con la información contenida en el manual, se desarrolla Ficha de Atención Personal (FAP) con sugerencias de adaptaciones curriculares y considerando la información recogida en proceso de orientación vocacional. Posteriormente, se capacita a docentes y jefes de carrera en Lengua de Señas Chilena e inclusión y Diseño Universal de Aprendizaje en concordancia con el nuevo Proyecto Educativo y Plan Estratégico Institucional.

- Modificación de infraestructura: considerando la normativa vigente se efectúan acciones de mejora de infraestructura considerando el principio de accesibilidad y diseño universal (instalación de ascensor, construcción de rampas, baños accesibles). 


\title{
4. Resultados
}

\begin{abstract}
A la luz de esta innovación educativa, en la tabla 1 y 2 se presentan los resultados obtenidos entre el 2017 y 2019, en cuanto a las actividades e intervenciones realizadas.
\end{abstract}

Tabla 1. Gestión de la instalación de una cultura inclusiva.

\begin{tabular}{lll}
\hline Línea & Acciones & Resultados \\
\hline & $\begin{array}{l}\text { Charlas de difusión a la comunidad } \\
\text { educativa }\end{array}$ & 38 charlas \\
\cline { 2 - 3 } & $\begin{array}{l}\text { Socialización con empresas e } \\
\text { instituciones de la región del Biobio y } \\
\text { Nuble sobre experiencia en temas de } \\
\text { inclusión }\end{array}$ & $\begin{array}{l}18 \text { empresas e } \\
\text { instituciones }\end{array}$ \\
\cline { 2 - 3 } $\begin{array}{l}\text { Gestión e } \\
\text { instalación de una } \\
\text { cultura inclusiva }\end{array}$ & $\begin{array}{l}\text { Proceso de admisión via Ley de } \\
\text { Inclusión 20.422 }\end{array}$ & 34 alumnos \\
\cline { 2 - 3 } & $\begin{array}{l}\text { Capacitación en lengua de señas } \\
\text { chilena }\end{array}$ & 41 orientaciones \\
\cline { 2 - 3 } & Modificación de infraestructura & $\begin{array}{l}\text { Accesibilidad de espacios } \\
\text { físicos }\end{array}$ \\
\hline
\end{tabular}

Tabla 2. Gestión académica.

\begin{tabular}{|c|c|c|}
\hline Línea & Acciones & Resultados \\
\hline \multirow{7}{*}{ Académica } & Manual de Atención a la Discapacidad & $100 \%$ docentes \\
\hline & $\begin{array}{l}\text { N. de estudiantes que cuentan con } \\
\text { Ficha de Atención Personal (FAP) }\end{array}$ & 23 estudiantes \\
\hline & $\begin{array}{l}\text { Docentes que aplican adaptaciones } \\
\text { curriculares no significativas }\end{array}$ & 124 docentes \\
\hline & Reuniones de seguimiento con alumnos & 72 reuniones \\
\hline & Formación transversal & $\begin{array}{l}100 \% \text { estudiantes } \\
\text { (con y sin discapacidad) }\end{array}$ \\
\hline & $\begin{array}{l}\text { Seminario Diseño Universal de } \\
\text { Aprendizaje }\end{array}$ & 67 personas \\
\hline & $\begin{array}{l}\text { Capacitación de diseño universal de } \\
\text { aprendizaje }\end{array}$ & 60 personas \\
\hline
\end{tabular}


Desde la implementación de las acciones del Programa de Inclusión, el año 2017 a la fecha, se han matriculado a nivel institucional 34 estudiantes con discapacidad física y sensorial, de los cuales se mantienen 27 con situación académica regular, correspondiente a un $79 \%$, y 2 se encuentran en situación de titulación oportuna. El promedio de notas del año 2017 es de 4,9 y del 2018 es de 5,4. Con respecto a la progresión académica, los estudiantes de la cohorte 2018 han aprobado el total de sus asignaturas, y la cohorte 2017 tiene un porcentaje de aprobación del $87 \%$. A partir de esta innovación, el instituto puede identificar a los estudiantes con discapacidad y generar los mecanismos de acompañamiento necesarios.

En un análisis cualitativo, podemos señalar que los resultados obtenidos son producto de la puesta en marcha de dos líneas de trabajo, que tienen como base el concepto de la corresponsabilidad, lo que significa mantener la autonomía, la libertad y generar igualdad en las oportunidades, eliminando las barreras para el aprendizaje y fomentando la participación activa, respondiendo a las necesidades de los estudiantes en situación de discapacidad, a las disposiciones legales, al plan estratégico institucional y proyecto educativo.

Los resultados se han obtenido a partir de la comprensión de que los cambios no se limitan solo a implementar procesos institucionales, sino también a educar a través de la concienciación de la comunidad tanto interna como externa, creando espacios reflexivos que permitan el diálogo abierto y respetuoso sobre la inclusión, favoreciendo el desarrollo de una cultura inclusiva de manera paulatina. Lo anterior ha generado transformaciones y compromisos a nivel institucional, ya que actualmente la temática de inclusión y diversidad es parte del Plan Estratégico Institucional 2018-2022 y también se ha incorporado al proyecto educativo.

Con el tiempo se ha observado que el conocimiento de la realidad de cada uno de los estudiantes ha permitido responder a sus experiencias previas, que tomen decisiones informados y que también sean responsables de su propio aprendizaje. 


\section{Conclusiones y discusión}

Nuestra institución busca que los estudiantes finalicen su proceso académico y no abandonen la carrera. Por ello, los resultados académicos señalados anteriormente responden a los indicadores institucionales y a los objetivos planteados que corresponden al diseño e implementación de un programa de inclusión integral, que los ayude a incorporarse y mantenerse en el nuevo sistema social, favoreciendo la progresión académica de los estudiantes y responda a los principios de respeto en la dignidad humana, autonomía, accesibilidad universal, no discriminación, diálogo civil, entre otros. Estamos trabajando con respecto a la diversidad, incorporando otras temáticas de inclusión, como género y etnias, a través de la adjudicación de varios proyectos, lo cual demuestra el compromiso de nuestra institución por seguir construyendo una cultura inclusiva.

\section{Referencias bibliográficas}

Armijo-Cabrera, M. (2018). Deconstruyendo la noción de inclusión: Un análisis de investigaciones, políticas y prácticas en educación. DOI: $<10.15359 /$ ree.22-3.8>.

Declaración de Salamanca (1994). Conferencia Mundial sobre Necesidades Educativas Especiales. Recuperado de: <http://paidos.rediris. es/genysi/recursos/doc/leyes/dec_sal.htm>.

Echeita, G.; Ainscow, M. (2011). «La educación inclusiva como derecho. Marco de referencia y pautas de acción para el desarrollo de una revolución pendiente». Tejuelo. Revista de Didáctica de la Lengua y la Literatura, IV: 26-45. Recuperado de: <http://iesgtballester.juntaextremadura.net/web/profesores/tejuelo/vinculos/articulos/r 12/03.pdf>.

Echeita, G.; Duk, C. (2008). «Inclusión educativa». Revista Iberoamericana sobre Calidad, Eficacia y Cambio en Educación, 6(2): 1-8. Recuperado de: <https://www.redalyc.org/pdf/551/55160201.pdf>.

Errandonea, M. (2016). Participación de personas en situación de discapacidad en el sistema de educación superior chileno (tesis para optar al grado de Magíster en Políticas Públicas). Universidad de Chile, Santiago.

Gobierno de Chile (2010). Ley 20.422. Establece normas sobre igualdad de oportunidades e inclusión social de personas con discapacidad. Biblioteca del Congreso Nacional de Chile. Recuperado 
de: <https://www.leychile.cl/Navegar?idNorma=1010903\&idParte $=8869259$ \&idVersion $=2010-02-10>$.

Gutiérrez-Ortega, M.; Martín-Cilleros, M. V.; Jenaro-Ríos, C. (2018). «La cultura, pieza clave para avanzar en los centros educativos». Revista de Educación Inclusiva, 11(2): 13-26. Recuperado de: <https://revista educacioninclusiva.es/index.php/REI/article/viewFile/325/355>.

Lissi, R.; Zuzulich, S.; Salinas, M.; Achiardi, C.; Hojas, A.; Pedrals, N. (2009). Discapacidad en contextos universitarios: Experiencia del Piane UC. DOI: <https://doi.org/10.31619/caledu.n30.183>.

Parra, C. (2011). «Educación inclusiva: Un modelo de diversidad humana». Revista Educación y Desarrollo Social, 5(1): 139-150. Recuperado de: <https://dialnet.unirioja.es/descarga/articulo/5386258.pdf>.

Paz, E. (2018). «La formación del profesorado universitario para la atención a la diversidad en la educación superior». IE Revista de investigación educativa de la Rediech, 9(16): 67-82. Recuperado de: <http:// www.scielo.org.mx/scielo.php?script=sci_arttext\&pid=S2448$85502018000100067 \& \operatorname{lng}=e s \& n r m=i s o>$.

Plancarte, P. (2017). «Inclusión educativa y cultura inclusiva». Revista Nacional e Internacional de Educación Inclusiva, 10(2): 213-226. Recuperado de: $<$ http://www.revistaeducacioninclusiva.es/index.php/ REI/article/view/294>.

SENADIS (Servicio Nacional de la Discapacidad) (2015). Segundo Estudio Nacional de la Discapacidad (ENDISC II). Santiago, Chile: SENADIS-Gobierno de Chile.

Unesco (2006). Informe sobre la educación superior en América Latina y el Caribe 2000-2005. La metamorfosis de la educación superior. Recuperado de: <https://www.inacap.cl/tportal/portales/tp4964b0e1bk102/ uploadImg/File/Educacion Superior/3_InfUNESCOEd_Sup_ AmLat2000-05.pdf>.

- (2009). Directrices sobre políticas de inclusión en la educación. Recuperado de: <http://unesdoc.unesco.org/images/0017/001778/177849s.pdf>.

Vásquez, B.; Alarcón, E. (2016). «La inclusión en las universidades chilenas: del discurso a las interacciones prácticas, políticas y culturales». Pensamiento Educativo. Revista de Investigación Educacional Latinoamericana, 53(2): 1-19. Recuperado de: <pensamientoeducativo. uc.cl/index.php/pel/article/download/876/1781>.

Victoria, J. (2013). El modelo social de la discapacidad: una cuestión de derechos humanos. DOI: <https://doi.org/10.5944/rduned.12.2013.11716>. 
1. La innovación educativa en el contexto de la

Educación Superior Técnico-Profesional.

Claudio Maregatti Solano, María luisa Arancibia Muñoz, Rosa Eliana Romero Alonso

1. Introducción

2. La transformación de las instituciones de

Educación Superior Técnico-Profesional

3. De las prácticas docentes al saber académico

4. El aporte al conocimiento científico sobre la

Educación Superior Técnico-Profesional . . . . . . . . . 15

5. Referencias bibliográficas .................. . 21

2. Caracterización de estudiantes de Educación Técnico-Profesional respecto a su Educación Secundaria: reflexiones para la Educación Superior

Javier Torres-Vallejos, Juan Ignacio Venegas Muggli, Simón Mundaca Toledo, Juan Carlos Oyanedel Sepúlveda

1. Introducción . . . . . . . . . . . . . . . . . . . 26

2. Metodología ......................... . 30

2.1 Muestra . . . . . . . . . . . . . . . . . . . . . 30

2.2 Variables . . . . . . . . . . . . . . . . . . . . . 32

2.3 Tratamiento de bases de datos . . . . . . . . . . . . 32

2.4 Análisis de datos..................... 33

3. Resultados ........................... 33

3.1 Características educativas y académicas en

Educación Superior ..................... . 34

3.2 Características educativas y académicas del establecimiento secundario de egreso de Enseñanza Media . . . . . . . . . . . . . . . . . . . . 36

4. Discusión y conclusiones. . . . . . . . . . . . . . . 37

5. Referencias bibliográficas ................. . . 39 
3. Aportes hacia la construcción de un perfil docente para el primer año: la importancia del vínculo empático, el valor académico y el trabajo colaborativo

Carlos Alberto Acevedo Cossio

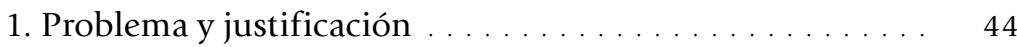

2. Marco teórico ....................... 45

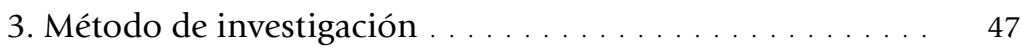

3.1 Diseño. . . . . . . . . . . . . . . . . . . 47

3.2 Participantes...................... 47

3.3 Técnicas e instrumentos de recolección de datos. . . . 48

3.4 Análisis de datos. . . . . . . . . . . . . . . . . . . . . 48

4. Resultados ... . . . . . . . . . . . . . . . . . . . . . . 49

4.1 Análisis de las entrevistas realizadas . . . . . . . . . 49

4.2 Análisis de cuestionarios . . . . . . . . . . . . . 50

4.2.1 Cuestionario de Estilo y Eficacia del Liderazgo . . 50

4.2.2 Cuestionario de Involucramiento Académico . . . 51

4.2.3 Índice de Reactividad Interpersonal . . . . . . . . . 52

5. Conclusiones y discusión ................. . 53

6. Referencias bibliográficas .................. 55

4. Mentoría docente para instalar estrategias de resolución de problemas en matemáticas iniciales

Cristian Iván Ramos Arrepol, Carlos Alberto Acevedo

Cossio

1. Problema y justificación . . . . . . . . . . . . . . . . 58

2. Marco teórico . . . . . . . . . . . . . . . . . . . . . . . 59

2.1 Trabajo colaborativo . . . . . . . . . . . . . . . . . 59

2.2 Mentorías ......................... 60

3. Método de investigación .................... 61

3.1 El perfil de entrada del docente . . . . . . . . . . . 62

3.2 Reclutamiento docente. . . . . . . . . . . . . 62

3.3 La caminata de aula . . . . . . . . . . . . . . . . . . 63

3.3.1 El primer paso . . . . . . . . . . . . . . . . 63

3.3 .2 Segunda ronda . . . . . . . . . . . . . . . . . . 64

3.3.3 Ronda final. . . . . . . . . . . . . . . . . . 64

3.4 Abordaje de situaciones críticas . . . . . . . . . . . . 64

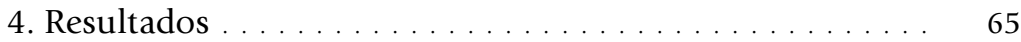

5. Conclusiones y discusión. . . . . . . . . . . . . . . . 69

6. Referencias bibliográficas ................... 70 
5. Clase invertida para la formación inicial de educadoras diferenciales sobre aprendizaje matemático

Laura Marjorie Espinoza Pastén

1. Problema y justificación . . . . . . . . . . . . . . . . . . 74

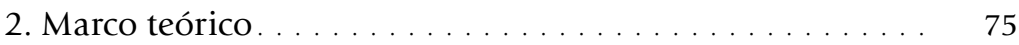

3. Proceso de la innovación. . . . . . . . . . . . . . . 76

3.1 Contexto ........................ 76

3.2 Diseño didáctico . . . . . . . . . . . . . . . 77

4. Resultados ......................... . 80

4.1 Resultados considerando el rendimiento académico. . 80

4.2 Resultados considerando la valoración de las estudiantes $\quad 81$

5. Discusión y conclusiones . . . . . . . . . . . . . . . 83

6. Referencias bibliográficas ................. 84

6. ¿Cómo medir el aprendizaje en innovación? Análisis factorial confirmatorio del Innovator's Behavior Questionnaire (i)BQ en universitarios chilenos. . . . . . 87 Jorge Maluenda Albornoz, Pedro Lledó Aninat

1. Problema y justificación . . . . . . . . . . . . . . . . . . . . . 89

2. Marco teórico . . . . . . . . . . . . . . . . . . . . . 90

3. Método............................. 92

4. Resultados ......................... 93

5. Conclusiones y discusión. . . . . . . . . . . . . . . . . 96

6. Referencias bibliográficas ................. 98

7. Programa de inclusión para actores de Educación Superior Técnico-Profesional . . . . . . . . . . . . . . . . 101

Marcela Pérez Poquet, Paulina Muñoz Villalobos, Victoria Aravena Rivas

1. Problema y justificación del estudio . . . . . . . . . . . . 102

2. Marco teórico . . . . . . . . . . . . . . . . . . . . . . 105

3. Metodología de investigación ................ 108

3.1 Descripción del contexto . . . . . . . . . . . . . . . 108

3.2 Participantes. . . . . . . . . . . . . . . . . . . . 109

3.3 Instrumentos y procedimientos . . . . . . . . . . . . . . 109

4. Resultados . . . . . . . . . . . . . . . . . . . . . . . 111

5. Conclusiones y discusión. . . . . . . . . . . . . . . . . . 113

6. Referencias bibliográficas ................. 113

Los autores. . . . . . . . . . . . . . . . . . . . . . . . . ${ }^{115}$ 